\title{
STRUCTURE OF RINGS SATISFYING CERTAIN IDENTITIES ON COMMUTATORS
}

\author{
MOHAN S. PUTCHA, ROBERT S. WILSON AND ADIL YAQUB
}

Abstract. Suppose $R$ is an associative ring with center $Z$, and suppose $J$ is the Jacobson radial of $R$. Suppose that, for all $x, y$ in $R$, there exist $z_{x, y} \in Z$ and an integer $n(x, y)>1$ such that

$$
x y-y x=(x y-y x)^{n(x, y)} z_{x, y} .
$$

Then $R / J$ is a subdirect sum of division rings satisfying: $(x y-y x)^{n(x, y)-1}$ is in the center. Additional results on the additive and multiplicative commutators which are in the center of a division ring satisfying (A) are also obtained. Next, suppose $D$ is a division ring of characteristic not 2 and with the property that, for some $x, y$ in $D$, there exists a positive integer $n$ such that $(x y-y x)^{n}$ is in the center, and suppose that the smallest such $n$ is even, then $D$ contains a subdivision ring isomorphic to the "generalized" quaternions (and conversely). Finally, it is proved that an arbitrary ring $R$ with the property that for all $x, y$ in $R$, there exists $z_{x, y}$ in $Z$ such that $x y-y x=(x y-y x)^{2} z_{x, y}$ is necessarily commutative, and that the exponent 2 cannot, in general, be replaced by 3 .

A well-known theorem of Herstein [1] asserts that if $R$ is an associative ring with the property that for all $x, y$ in $R$, there exists an integer $n(x, y)>1$ such that $(x y-y x)^{n(x, y)}=x y-y x$, then $R$ is commutative. Our present object is to investigate the structure of an associative ring $R$ with the property that, for all $x, y$ in $R$, there exists an element $z_{x, y}$ and an integer $n(x, y)>1$ such that $z_{x, y}$ is in the center $Z$ of $R$ and

$$
x y-y x=(x y-y x)^{n(x, y)} z_{x, y} .
$$

Indeed, we show that for such a ring $R, R / J$ is isomorphic to a subdirect sum of division rings satisfying: $(x y-y x)^{n(x, y)-1}$ is in the center, where $J$ denotes the Jacobson radical of $R$. This, in turn, raises the question: what can be said about a division ring $D$ in which some power of every commutator $x y-y x$ is in the center $Z$ ? In this connection, we prove that such a division ring $D$ has the property that every multiplicative commutator $x y x^{-1} y^{-1}$ which is in the center of $D$ is necessarily an $m$ th root

Received by the editors October 26, 1970 and, in revised form, March 17, 1971. AMS 1970 subject classifications. Primary 16A70, 16A38; Secondary 16A48. 
of unity (for some $m$ ). We also show that if the division ring $D$ satisfies: $(x y-y x)^{n(x, y)}$ is in the center $Z$ of $D$, and if, in addition, $n(x, y)$ is prime to the characteristic of $D$, then every additive commutator $x y-y x$ in $Z$ is necessarily zero. We then consider the case in which the division ring $D$ has the property that, for some $x, y$ in $D$, there exists a positive integer $n$ such that $(x y-y x)^{n}$ is in the center, and in which the smallest such $n$ is even. For such a division ring $D$, we show that $D$ contains a subdivision ring isomorphic to a division ring of "generalized" quaternions. Finally, we prove that an arbitrary ring $R$ with the property that, for all $x, y$ in $R$, there exists $z_{x, y}$ in $Z$ such that $x y-y x=(x y-y x)^{2} z_{x, y}$ is necessarily commutative, and that this theorem is, in general, not true if the exponent 2 is replaced by an arbitrary integer.

In preparation for the proof of the main theorems, we first establish the following lemmas.

LEMMA 1. Suppose $R$ is an associative ring with center $Z$, and suppose $J$ and $C(R)$ denote the Jacobson radical and commutator ideal of $R$, respectively. Suppose that, for all $x, y$ in $R$, there exist an element $z=z_{x, y}$ (depending on $x$ and $y$ ), $z_{x, y} \in Z$, and an integer $n=n(x, y)>1$, such that

$$
x y-y x=(x y-y x)^{n} z_{x, y} .
$$

Then (i) $J \subseteq Z$; (ii) $J C(R)=C(R) J=(0)$; (iii) all the idempotents of $R$ are in the center of $R$.

Proof. Suppose $x \in J, y \in R$. By (1), $(x y-y x)^{n-1} z_{x, y}$ is an idempotent element (since $z_{x, y} \in Z$ ) in $J$, and hence $(x y-y x)^{n-1} z_{x, y}=0$, since $J$ contains no nonzero idempotents. Therefore, by (1), $x y-y x=0$, and (i) is proved.

To prove (ii), suppose that $a \in J$ and $x, y \in R$. Then, using the fact that $J \subseteq Z$, we get

$$
\begin{aligned}
a(x y-y x) & =a x y-(a y) x=a x y-x(a y) \\
& =a x y-x(y a)=a(x y)-(x y) a=0 .
\end{aligned}
$$

Hence $a C(R)=C(R) a=(0)$ for all $a \in J$, and (ii) follows at once.

To prove (iii), suppose $e^{2}=e \in R$, and suppose $a \in R$. Then, $(e a-e a e)^{2}=0$. Moreover, by (1),

$$
e(e a-e a e)-(e a-e a e) e=\{e(e a-e a e)-(e a-e a e) e\}^{n} z=0,
$$

and hence $e a=e a e$. A similar argument shows that $a e=e a e$. Thus $e a=a e$ for all $a$ in $R$, and the lemma is proved.

LEMMA 2. Suppose $R$ is an associative ring which satisfies the hypotheses of Lemma 1. Suppose, further, that the intersection of any two nonzero ideals in $R$ is nonzero. Then (i) the only idempotents in $R$ are 0 and 1 ; (ii) if $x y-y x \neq 0$, then $(x y-y x)^{n-1} \in Z$ and is invertible. 
Proof. Suppose $e \in R, e^{2}=e$, and define $A$ and $B$ by

$$
A=\{e x-x \mid x \in R\}, \quad B=\{e x \mid x \in R\} .
$$

Since, by Lemma $1, e$ is in the center of $R$, it is easily seen that both $A$ and $B$ are ideals in $R$, and, moreover, $A \cap B=(0)$. Hence, by hypothesis, $A=(0)$ or $B=(0)$. Now, if $A=(0)$, then $x e=e x=x$ for all $x$ in $R$, and hence $e=1$. On the other hand, if $B=(0)$, then $e=e e=0$. This proves (i).

Next, we proceed to prove (ii). Thus suppose $x, y \in R$, and $x y-y x \neq 0$. Since $z_{x, y}$ is in the center, we conclude from (1) that $(x y-y x)^{n-1} z_{x, y}$ is idempotent and hence, for all $x, y$ in $R$,

$$
(x y-y x)^{n-1} z_{x, y}=0 \text { or }(x y-y x)^{n-1} z_{x, y}=1 .
$$

Now, if $(x y-y x)^{n-1} z_{x, y}=0$, then by (1), $x y-y x=(x y-y x)^{n} z_{x, y}=0$, a contradiction. Hence $(x y-y x)^{n-1} z_{x, y} \neq 0$, and therefore by (2), we have $(x y-y x)^{n-1} z_{x, y}=1$. Since $z_{x, y} \in Z$, we get

$$
(x y-y x)^{n-1} z_{x, y}=z_{x, y}(x y-y x)^{n-1}=1,
$$

and hence $(x y-y x)^{n-1}=z_{x, y}^{-1} \in Z$ (since $z_{x, y} \in Z$ ). Thus (ii) follows, and the lemma is proved.

As an immediate consequence of Lemma 2, we have the following

COROLlary 1. Suppose $R$ is a prime ring or a subdirectly irreducible ring which satisfies the hypotheses of Lemma 1 . Then (i) the only idempotents in $R$ are 0 and 1 ; (ii) $x y-y x \neq 0$ implies that $(x y-y x)^{n-1} \in Z$ and is invertible.

LEMMA 3. Suppose $R$ is an associative ring which satisfies the hypotheses of Lemma 1. If, further, $R$ is primitive, then $R$ is a division ring.

Proof. By the Jacobson Density Theorem, if $R$ is not a division ring, then for any $n$, there is a subring of $R$ with homomorphic image the ring $D_{n}$ of $n \times n$ matrices over a division ring $D$. Moreover, (1) is preserved in $D_{n}$, and hence by Corollary 1 , the prime ring $D_{n}$ cannot have any nontrivial idempotents, a contradiction. This contradiction proves the lemma.

As an immediate consequence of Lemma 3, we have

THEOREM 1. In the notation, and under all the hypotheses, of Lemma 1, we have that $R / J$ is isomorphic to a subdirect sum of division rings satisfying $(x y-y x)^{n-1}$ is in the center.

Proof. Since $R / J$ is semisimple, it is isomorphic to a subdirect sum of primitive rings $[2$, p. 14] each of which clearly satisfies the hypotheses of the theorem. The theorem now follows at once from Lemma 3.

In view of Theorem 1, it is quite natural to ask what can be said about a division ring in which some power of every commutator is in the center. In the next theorems we give some results in this direction. 
THEOREM 2. Let $D$ be a division ring with the property that, for all $x, y$ in $D$, there exists a positive integer $n(x, y)$ such that $(x y-y x)^{n(x, y)}$ is in the center $Z$ of $D$. Let $c$ be any multiplicative commutator which is in the center $Z$. Then, for some positive integer $m, c^{m}=1$.

Proof. Let $c=x y x^{-1} y^{-1}$, where $c \in Z$. Then $x y=c y x$. Now, by hypothesis, the commutator $x\left(x^{-1} y\right)-\left(x^{-1} y\right) x=y-x^{-1} y x$ satisfies

$$
\left(y-x^{-1} y x\right)^{m} \in Z \text {, for some positive integer } m=n\left(x, x^{-1} y\right) \text {. }
$$

Hence, $\left(y-x^{-1}\left(c^{-1} x y\right)\right)^{m} \in Z$, and thus $\left(y-y c^{-1}\right)^{m} \in Z$ (since $c^{-1} \in Z$ ). This reduces to $y^{m}\left(1-c^{-1}\right)^{m} \in Z$. Now, if $c^{-1}=1$, then we are clearly done. Thus suppose $c^{-1} \neq 1$. Since $\left(1-c^{-1}\right) \in Z$ and $y^{m}\left(1-c^{-1}\right)^{m} \in Z$, we get $y^{m} \in Z$. Combining this with $x y=c y x, c \in Z$, we obtain $x y^{m}=c^{m} y^{m} x=c^{m} x y^{m}$, and hence $c^{m}=1$. This proves the theorem.

THEOREM 3. Let $D$ be a division ring of characteristic $q$ and with the property that, for all $x, y$ in $D$, there exists a positive integer $n(x, y)$ such that $(x y-y x)^{n(x, y)}$ is in the center $Z$ of $D$. If, further, $n(x, y)$ is prime to $q$, then every additive commutator in $Z$ is zero.

Proof. Let $x, y \in D$ and let $[x, y]=x y-y x$. Suppose $[x, y] \in Z$ and $[x, y] \neq 0$. We shall show that this leads to a contradiction. First, observe that $x[x, y]=[x y,-x]$ and hence $x[x, y]$ is a commutator. Therefore, by hypothesis, there exists a positive integer $n=n(x y,-x)$ prime to the characteristic, such that $(x[x, y])^{n} \in Z$. Since $[x, y] \in Z$, we obtain $x^{n}[x, y]^{n} \in Z$. Again, since $[x, y] \in Z$ and $[x, y] \neq 0$, we get $[x, y]^{-n} \in Z$, and hence $\left(x^{n}[x, y]^{n}\right)[x, y]^{-n} \in Z$, that is $x^{n} \in Z$. Now, since $[x, y]$ commutes with $x$, an easy induction yields $[2$, p. 221]

$$
0=\left[x^{n}, y\right]=n x^{n-1}[x, y]
$$

and hence $n \cdot 1=0$, since $[x, y] \neq 0$. This contradicts the hypothesis that $n$ is prime to the characteristic of $D$, and the theorem is proved.

Observe that if the division ring $D$ has characteristic zero, then the hypothesis " $n(x, y)$ is prime to $q$ " is not needed in Theorem 3. In particular, we have

COROLlaRY 2. The ring of quaternions has no nonzero additive commutators in the center.

Proof. This follows at once from Theorem 3, upon observing that the quaternions satisfy: $(x y-y x)^{2} \in Z$.

By a generalized quaternion algebra over a field $Z$ of characteristic not 2, we mean an algebra over $Z$, generated by two elements $\alpha, \beta$ not in $Z$, subject to the relations

$$
\alpha \beta=-\beta \alpha, \quad \alpha^{2} \in Z, \quad \beta^{2} \in Z .
$$


(See [4, p. 142].) As is well known [4, §57.2], a quaternion algebra over $Z$ is central simple over $Z$.

Our final result on division rings is the following

THEOREM 4. Let $D$ be a division ring of characteristic not 2 and with center $Z$. Suppose that, for some $x, y$ in $D$, there exists a positive integer $n$ such that $(x y-y x)^{n}$ is in $Z$. If, further, the smallest such $n$ is even, then $D$ contains a subdivision ring $D_{1}$ which is isomorphic to a generalized quaternion division algebra over some extension field of $Z$.

Proof. Let $x, y \in D$ and let $n$ be the least positive integer such that $(x y-y x)^{n}$ is in the center $Z$ of $D$. Let $n=2^{k} m, k \geqq 1, m$ odd. By the minimality of $n$, there exists a commutator $b$ in $D$ such that $b^{n} \in Z$, $b^{n / 2} \notin Z$. Let $\alpha=b^{n / 2}$. Since $\alpha \notin Z, \alpha y_{0} \neq y_{0} \alpha$ for some element $y_{0}$ in $D$. Moreover, since $\alpha^{2} \in Z$, we have

$$
\alpha\left(\alpha y_{0}-y_{0} \alpha\right)=-\left(\alpha y_{0}-y_{0} \alpha\right) \alpha .
$$

Let $\beta=\left(\alpha y_{0}-y_{0} \alpha\right)^{m}$. Since $m$ is odd, (5) readily implies that $\alpha \beta=-\beta \alpha$. In addition, since $\alpha \beta=-\beta \alpha$, we have $\alpha \beta^{2}=\beta^{2} \alpha$. Now, let $D_{1}=Z(\alpha, \beta)$, and let $Z_{1}$ be the center of the subdivision ring $D_{1}$. Then, as we have just shown,

$$
\alpha \beta=-\beta \alpha, \quad \alpha^{2} \in Z_{1} \text { (since } \alpha^{2} \in Z \text { ), and } \beta^{2} \in Z_{1} \text { (since } \beta^{2} \alpha=\alpha \beta^{2} \text { ). }
$$

Thus (see (4)), $D_{1}$ is isomorphic to a generalized quaternion algebra over $Z_{1}$, and the theorem is proved.

Our next result is concerned with the special case which arises upon taking $n=2$ in (1). In this connection, we have the following

THEOREM 5. Suppose $R$ is an associative ring with center $Z$, and suppose that for all $x, y$ in $R$, there exists an element $z_{x, y}$ (depending on $x$ and $y$ ), $z_{x, y} \in Z$, such that

$$
x y-y x=(x y-y x)^{2} z_{x, y} .
$$

Then $R$ is commutative (and conversely).

Proof. Let $J$ be the Jacobson radical of $R$. Now, by Theorem 1 (with $n=2), R / J$ is isomorphic to a subdirect sum of division rings $D_{i}, i \in \Gamma$, where each $D_{i}$ satisfies: $x y-y x$ is in the center. Hence, by Theorem 3 (with $n(x, y)=1$ ), we get $x y-y x=0$ for all $x, y \in D_{i}$. Therefore, $R / J$ is isomorphic to a subdirect sum of fields, and is thus commutative. Hence $x y-y x \in J$, for all $x, y$ in $R$. Now, since $z_{x, y} \in Z$, we get, using (6), $(x y-y x) z_{x, y}$ is an idempotent element in $J$, and hence $(x y-y x) z_{x, y}=0$. Hence, by (6), $x y-y x=0$ for all $x, y$ in $R$, and the theorem is proved. 
It is noteworthy to observe that any generalized quaternion division algebra satisfies " $(x y-y x)^{2} \in Z$ " and hence $x y-y x=(x y-y x)^{3} z_{x, y}$, for some $z_{x, y} \in Z$. However, such a ring is not commutative. Thus Theorem 5 need not be true when " $(x y-y x)^{2}$ " is replaced by " $(x y-y x)^{3}$ ". In addition, this shows that the converse of Theorem 4 is also true.

In conclusion, we wish to express our indebtedness and gratitude to the referee for his valuable suggestions.

\section{REFERENCES}

1. I. N. Herstein, A condition for the commutativity of rings, Canad. J. Math. 9 (1957), 583-586. MR 19, 1035.

2. N. Jacobson, Structure of rings, 2nd ed., Amer. Math. Soc. Colloq. Publ., vol. 37, Amer. Math. Soc., Providence, R.I., 1964. MR 36 \#5158.

3. N. H. McCoy, The theory of rings, Macmillan, New York, 1964. MR 32 \#5680.

4. O. T. O'Meara, Introduction to quadratic forms, Die Grundlehren der math. Wissenschaften, Band 117, Academic Press, New York; Springer-Verlag, Berlin, 1963. MR 27 \#2485.

Department of Mathematics, University of California, Santa Barbara, California 93106 